\title{
An expedient synthesis of thienylacetic acids using the Willgerodt-Kindler reaction under PTC conditions
}

\author{
Vitaly A. Podshibyakin, ${ }^{\text {a }}$ Evgenii N. Shepelenko, ${ }^{\text {b }}$ Karina S. Tikhomirova, ${ }^{\text {a }}$ \\ Alexander D. Dubonosov, ${ }^{* b}$ Vladimir A. Bren, ${ }^{a}$ and Vladimir I. Minkin ${ }^{\text {a,b }}$ \\ ${ }^{a}$ Institute of Physical and Organic Chemistry, Southern Federal University, 194/2, Stachka Av., \\ 344090 Rostov on Don, Russian Federation \\ ${ }^{b}$ Southern Scientific Center of Russian Academy of Sciences,41, Chekhov Pr., \\ 344006 Rostov on Don, Russian Federation \\ E-mail: aled@ipoc.sfedu.ru
}

DOI: https://doi.org/10.24820/ark.5550190.p009.901

\begin{abstract}
Novel (5-aryl-2-methylthiophen-3-yl)acetic acids were synthesized starting from 3-aryl-3chloroacrylaldehydes via corresponding thienylcarbaldehydes and thienylethanones using Willgerodt-Kindler reaction under phase-transfer conditions. Their structures were established based on the data of ${ }^{1} \mathrm{H},{ }^{13} \mathrm{C}$ NMR, IR spectroscopy and mass-spectrometry.
\end{abstract}

Keywords: 3-Thienylacetic acids, Willgerodt-Kindler reaction, phase-transfer catalysis

\section{Introduction}

Derivatives of 3-thienylacetic acids are important intermediates in the synthesis of drugs, ${ }^{1}$ pesticides $^{2}$ and multifunctional photochromic molecular systems possessing fluorescent, ${ }^{3-5}$ magnetic $^{6}$ and complexing properties. ${ }^{7}$ The basic methods used for the synthesis of these compounds are based on hydrolysis of the corresponding nitriles, ${ }^{8}$ the reduction of ketoacids, ${ }^{9}$ Arndt-Eistert ${ }^{10}$ and Willgerodt-Kindler ${ }^{11}$ reactions. The Willgerodt-Kindler reaction is usually applied for the preparation of (thio)amides, carboxylic acids, and heterocycles. ${ }^{12}$ At the same time because of the low yields of the targeted compounds and formation of complex reaction mixtures ${ }^{13}$ this reaction has not been more widely employed in organic synthesis. Only a few papers have been published on application of this method for the synthesis of arylacetic acids performed under conditions of the phase-transfer catalysts (PTC). ${ }^{14}$ Herein we report an expedient procedure for the synthesis of 5-arylsubstituted 3-thienylacetic acids starting from 3-aryl-3-chloroacrylaldehydes via corresponding thienylcarbaldehydes and thienylethanones based on the Willgerodt-Kindler reaction under PTC conditions. 


\section{Results and Discussion}

No convenient and unequivocally characterized method for the preparation of 5-arylthiophene-2carbaldehydes has yet been developed. ${ }^{15-19}$ Aldehyde 1a was previously synthesized by the Vilsmeier formylation of 5-(4-chlorophenyl)thiophene, ${ }^{16}$ but no spectral data on the prepared compound were presented. The usually used Suzuki cross-coupling reaction of thienylboronic acids with bromothienyl compounds requires an inert gas atmosphere, palladium catalyst $\operatorname{Pd}\left(\mathrm{PPh}_{3}\right)_{4}$ and takes a long reaction time. ${ }^{20}$ Thus, this reaction for the synthesis of aldehyde $\mathbf{1 b}$ proceeds in $47 \mathrm{~h}$ (yield 31\%). Our approach to the synthesis of 5-arylthiophene-2-carbaldehydes involves interaction of 3-chloro-3-arylacrylaldehydes with sodium sulfide and chloroacetaldehyde in DMF to give the compounds 1a,b in 44-59\% yields (Scheme 1). The reaction time of the general procedure for preparation of $\mathbf{1 a}, \mathbf{b}$ has been shortened to 6.5 hours.

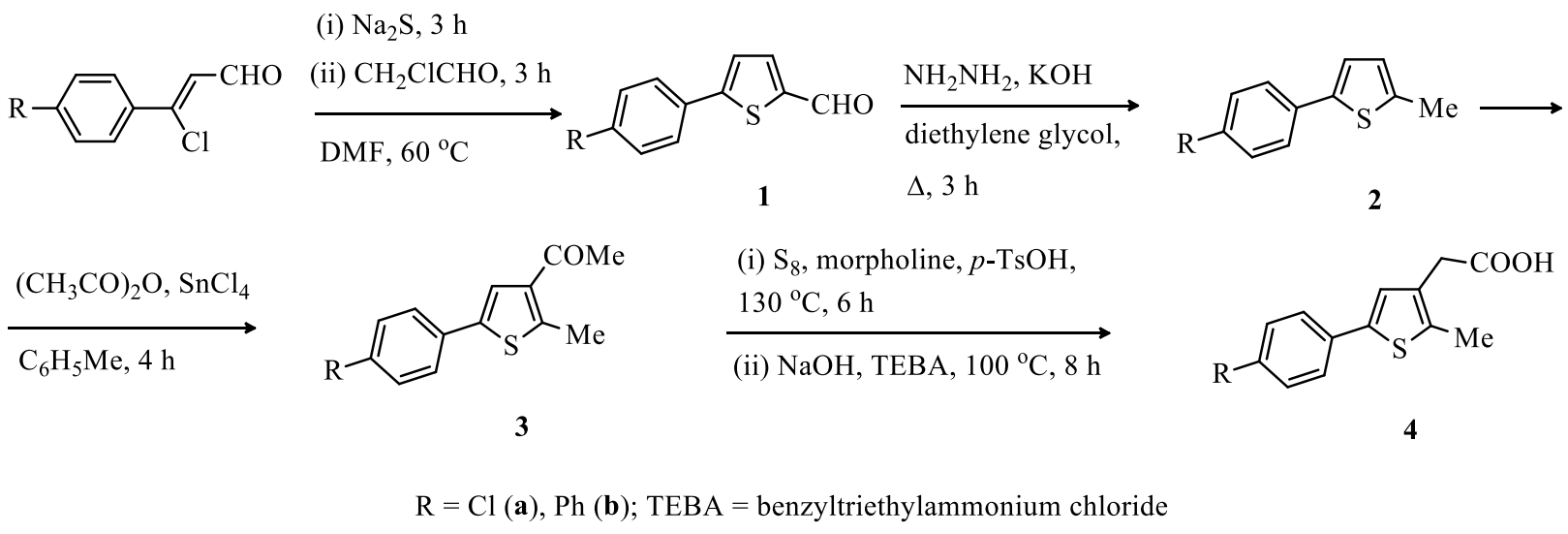

Scheme 1. Synthesis of 3-thienylacetic acids $\mathbf{4 a , b .}$

2-Methyl-5-(4-chlorophenyl)thiophene 2a was previously obtained by the treatment of 2methyl-5-(4-chlorophenyl)furan with hydrogen sulfide under conditions of acid catalysis. ${ }^{21}$ The reaction time was $48.5 \mathrm{~h}$ and the yield $54 \%$. A significant drawback of this reaction is the presence in the reaction mixture of products of hydrolytic cleavage of the furans. We reduced aldehydes 1a,b by hydrazine hydrate via Kishner-Wolff reaction which led to 2-methyl-5-arylthiophenes 2a,b in 30-40\% yields after $3 \mathrm{~h}$ reflux of diethylene glycol solution. Acylation of 2a,b with acetic anhydride in toluene in the presence of $\mathrm{SnCl}_{4}$ gave rise to 1-(2-methyl-5-(arylthiophen-3yl)ethanones 3a,b in $41-84 \%$ yields. Then these compounds were exposed to the WillgerodtKindler reaction with sulfur and morpholine followed by the treatment of aqueous sodium hydroxide in the presence of a phase-transfer catalyst (benzyltriethylammonium chloride, TEBA) which produced 2-(2-methyl-5-(4-chlorophenyl)thiophen-3-yl)acetic acids $\mathbf{4 a , b}$ in moderate 53$64 \%$ yields. The described synthetic protocol (Scheme 1) allows preparation of various functionalized 5-aryl substituted thienylacetic acids starting from the corresponding 3-aryl-3chloroacrylaldehydes. 
To evaluate the effectiveness of the Willgerodt-Kindler reaction under PTC conditions, we synthesized the known 2-(2,5-dimethylthiophen-3-yl)acetic acid (4c) and 2-(5-(4-bromophenyl)2-methylthiophen-3-yl)acetic acid (4d). These compounds were previously obtained from 1-(2,5dimethylthiophen-3-yl)ethanone ${ }^{22}$ and 1-(5-(4-chlorophenyl)-2-methylthiophen-3-yl)ethanone, ${ }^{13}$ correspondingly, in two steps including isolation of 2-(2,5-dimethylthiophen-3-yl)-1morpholinoethanethione and 2-(5-(4-bromophenyl)-2-methylthiophen-3-yl)-1-morpholinoethanethione and there subsequent hydrolysis. The above-described one-pot synthetic procedure led to acids $\mathbf{4 c}$ and $\mathbf{4 d}$ in good yields. The structures of the synthesized compounds $\mathbf{1 - 4}$ were confirmed by the data of ${ }^{1} \mathrm{H},{ }^{13} \mathrm{C}$ NMR and IR spectra.

\section{Conclusions}

We report on the expedient synthetic protocol which allows to prepare various novel 5arylsubstituted 3-thienylacetic acids starting from 3-aryl-3-chloroacrylaldehydes via corresponding thienylcarbaldehydes and thienylethanones under phase-transfer conditions.

\section{Experimental Section}

General. The ${ }^{1} \mathrm{H}$ and ${ }^{13} \mathrm{C}$ NMR spectra in $\mathrm{CDCl}_{3}$ were recorded on a Bruker DPX-250 (250 MHz for ${ }^{1} \mathrm{H}, 62.9 \mathrm{MHz}$ for $13 \mathrm{C}$ ) spectrometer, the signals were referred with respect to the signals of residual protons of deutero-solvent (7.24 ppm), $\delta$ values were measured with precision $0.01 \mathrm{ppm}$. The IR spectra were recorded on a Varian Excalibur 3100 FT-IR instrument using the attenuated total internal reflection technique (ZnSe crystal). Mass spectra were recorded on a Shimadzu GCMS-QP2010SE instrument with direct sample entry into the ion source (EI, $70 \mathrm{eV}$ ). Elemental analysis was performed on a KOVO CHN-analyzer. Melting points were determined on a PTP (M) instrument.

General procedure for the synthesis of 1a,b. A solution of 3-chloro-3-(4chlorophenyl)acrylaldehyde (32 mmol, $6.5 \mathrm{~g}$ ) or 3-([1,1'-biphenyl]-4-yl)-3-chloroacrylaldehyde (32 mmol, $7.8 \mathrm{~g}$ ) in $100 \mathrm{~mL}$ of dry DMF was added dropwise with stirring to a suspension of $\mathrm{Na}_{2} \mathrm{~S} \cdot 9 \mathrm{H}_{2} \mathrm{O}(33 \mathrm{mmol}, 7.9 \mathrm{~g})$ in $40 \mathrm{~mL}$ of dry DMF at $60{ }^{\circ} \mathrm{C}$ within $1 \mathrm{~h}$. The reaction mixture was stirred for $2 \mathrm{~h}$ at $60{ }^{\circ} \mathrm{C} .50 \%$ aqueous chloroacetaldehyde $(36 \mathrm{mmol}, 6 \mathrm{~mL})$ was added dropwise and the reaction mixture was stirred for $3 \mathrm{~h}$ at $60{ }^{\circ} \mathrm{C}$. A solution of $\mathrm{K}_{2} \mathrm{CO}_{3}(60 \mathrm{mmol}, 8.3 \mathrm{~g})$ in 10 $\mathrm{mL}$ of water was added and the stirring at the same temperature was continued for $0.5 \mathrm{~h}$. The reaction mixture was then poured into $1000 \mathrm{~mL}$ of water. The precipitate was filtered, washed with water, dried and recrystallized from ethanol with the charcoal.

5-(4-Chlorophenyl)thiophene-2-carbaldehyde (1a). Yield $4.2 \mathrm{~g}$ (59\%), light yellow solid, mp 88-89 ${ }^{\circ} \mathrm{C}$ [lit. $\left.{ }^{16} \mathrm{mp} 87{ }^{\circ} \mathrm{C}\right]$. IR $\left(v_{\max }, \mathrm{cm}^{-1}\right): 1655(\mathrm{C}=\mathrm{O}), 1599(\mathrm{C}=\mathrm{C}), 1491,1431 .{ }^{1} \mathrm{H}$ NMR $(250$ 
$\left.\mathrm{MHz}, \mathrm{CDCl}_{3}\right): \delta 7.36-7.40(\mathrm{~m}, 3 \mathrm{H}), 7.59(\mathrm{~d}, J 8.40 \mathrm{~Hz}, 2 \mathrm{H}), 7.73(\mathrm{~d}, J 3.80 \mathrm{~Hz}, 1 \mathrm{H}), 9.88(\mathrm{~s}, 1 \mathrm{H}$, CHO). EIMS, $70 \mathrm{eV}, \mathrm{m} / z: 222[\mathrm{M}]^{+}$. Anal. Calcd. for $\mathrm{C}_{11} \mathrm{H}_{7} \mathrm{ClOS}$ : C, 59.33; H, 3.17. Found: C, $59.23 ; \mathrm{H}, 3.15 \%$.

5-([1,1'-Biphenyl]-4-yl)thiophene-2-carbaldehyde (1b). Yield $3.7 \mathrm{~g}$ (44\%), light yellow solid, mp 194-195 ${ }^{\circ} \mathrm{C}$ [lit. $\left.{ }^{19} \mathrm{mp} 193-194{ }^{\circ} \mathrm{C}\right]$. IR $\left(v_{\max }, \mathrm{cm}^{-1}\right): 1664(\mathrm{C}=\mathrm{O}), 1608(\mathrm{C}=\mathrm{C}) .{ }^{1} \mathrm{H}$ NMR $(250$ $\mathrm{MHz}, \mathrm{CDCl}_{3}$ ): $\delta 7.32-7.85(\mathrm{~m}, 11 \mathrm{H}$, arom. H, thioph. H), 9.92 (s, $1 \mathrm{H}, \mathrm{CHO})$. MS (EI, $\left.70 \mathrm{eV}\right), \mathrm{m} / z$ : $264[\mathrm{M}]^{+}$. Anal. Calcd. for $\mathrm{C}_{17} \mathrm{H}_{12} \mathrm{OS}: \mathrm{C}, 77.24 ; \mathrm{H}, 4.58$. Found: C, 77.41; H, $4.35 \%$.

General procedure for the synthesis of $\mathbf{2 a}, \mathbf{b}$. A mixture of $\mathbf{1 a}(10 \mathrm{mmol}, 2.2 \mathrm{~g})$ or $\mathbf{1 b}(10 \mathrm{mmol}$, $2.6 \mathrm{~g}), 85 \%$ hydrazine hydrate $(135 \mathrm{mmol}, 6.75 \mathrm{~g})$ and $\mathrm{KOH}(75 \mathrm{mmol}, 4.2 \mathrm{~g})$ in $50 \mathrm{~mL}$ of diethylene glycol was stirred at reflux for $3 \mathrm{~h}$. The solution was then diluted with water $(150 \mathrm{~mL})$. The crude product was filtered, dried and recrystallized from ethanol.

2-(4-Chlorophenyl)-5-methylthiophene (2a). Yield $0.84 \mathrm{~g}$ (40\%), light yellow solid, mp 107$109{ }^{\circ} \mathrm{C}$ [lit. $\left.{ }^{20} \mathrm{mp} 108-109{ }^{\circ} \mathrm{C}\right]$. IR ( $\left.v_{\max }, \mathrm{cm}^{-1}\right): 1599,1491,1431 .{ }^{1} \mathrm{H}$ NMR $\left(250 \mathrm{MHz}, \mathrm{CDCl}_{3}\right): \delta$ 2.54 (s, 3H, Me), 6.68-7.50 (m, 6H, arom. H, thioph. H). MS (EI, $70 \mathrm{eV}), \mathrm{m} / z: 208[\mathrm{M}]^{+}$. Anal. Calcd. for $\mathrm{C}_{11} \mathrm{H}_{9} \mathrm{ClS}$ : C, 63.30; H, 4.35. Found: C, 63.43; H, $4.45 \%$.

2-([1,1'-Biphenyl]-4-yl)-5-methylthiophene (2b). Yield $0.75 \mathrm{~g}$ (30\%), light yellow solid, $\mathrm{mp}$ 144-145 ${ }^{\circ} \mathrm{C}$. IR $\left(v_{\max }, \mathrm{cm}^{-1}\right): 3058,1598,1497 .{ }^{1} \mathrm{H}$ NMR $\left(250 \mathrm{MHz}, \mathrm{CDCl}_{3}\right): \delta 2.54(\mathrm{~s}, 3 \mathrm{H}, \mathrm{Me})$, 6.84-6.86 (m, 1H, thioph. H), 7.32-7.74 (m, 10H, arom. H). $\left.{ }^{13} \mathrm{C} \mathrm{NMR} \mathrm{(62.9} \mathrm{MHz,} \mathrm{CDCl}_{3}\right): \delta 17.15$; $124.95 ; 127.58 ; 127.59 ; 128.30 ; 128.67 ; 128.68 ; 128.99 ; 129.30 ; 129.31 ; 130.75 ; 130.76 ; 135.62$; 141.51; 141.76; 142.34; 143.31. MS (EI, $70 \mathrm{eV}), \mathrm{m} / z: 250[\mathrm{M}]^{+}$. Anal. Calcd. for $\mathrm{C}_{17} \mathrm{H}_{14} \mathrm{~S}: \mathrm{C}$, 81.56; H, 5.64. Found: C, 81.43; H, $5.49 \%$.

General procedure for the synthesis of 3a,b. A solution of $\mathbf{2 a}(10 \mathrm{mmol}, 2.1 \mathrm{~g})$ or $\mathbf{2 b}(10 \mathrm{mmol}$, $2.5 \mathrm{~g}$ ) in $75 \mathrm{~mL}$ of dry toluene was cooled to $0-5{ }^{\circ} \mathrm{C}$. Acetic anhydride ( $20 \mathrm{mmol}, 2.0 \mathrm{~g}$ ) and then $\mathrm{SnCl}_{4}(20 \mathrm{mmol}, 5.2 \mathrm{~g}$ ) were added under stirring at this temperature. The reaction mixture was heated to $20^{\circ} \mathrm{C}$, stirred for $4 \mathrm{~h}$ and then acidified with $10 \%$ aqueous $\mathrm{HCl}(100 \mathrm{~mL})$. The organic layer was separated and dried $\left(\mathrm{Na}_{2} \mathrm{SO}_{4}\right)$. The solvent was removed using a rotary evaporator to give a crude residue which was recrystallized from ethanol.

1-(5-(4-Chlorophenyl)-2-methylthiophen-3-yl)ethanone (3a). Yield $1.0 \mathrm{~g} \mathrm{(41 \% ),} \mathrm{light} \mathrm{yellow}$ solid, mp 114-115 ${ }^{\circ} \mathrm{C}$. IR ( $\left.v_{\max }, \mathrm{cm}^{-1}\right): 1800(\mathrm{C}=\mathrm{O}), 1750(\mathrm{C}=\mathrm{C}), 1431 .{ }^{1} \mathrm{H}$ NMR $(250 \mathrm{MHz}$, $\left.\mathrm{CDCl}_{3}\right): \delta 2.51$ (s, 3H, Me), 2.73 (s, 3H, Me), 7.24 (s, 1H, thioph. H), 7.31-7.34 (d, 2H, arom. H), 7.44-7.47 (d, 2H, arom. H). ${ }^{13} \mathrm{C} \mathrm{NMR}\left(62.9 \mathrm{MHz}, \mathrm{CDCl}_{3}\right): \delta 16.30 ; 29.82 ; 124.60 ; 124.61 ; 126.78$; $126.79 ; 129.15 ; 129.16 ; 133.56 ; 137.04 ; 138.15 ; 148.73 ; 193.88 . \mathrm{MS}(\mathrm{EI}, 70 \mathrm{eV}), \mathrm{m} / z: 250$ [M] $^{+}$. Anal. Calcd. for $\mathrm{C}_{13} \mathrm{H}_{11} \mathrm{ClOS}$ : C, 62.27; H, 4.42. Found: C, 62.34; H, $4.45 \%$.

1-(5-(1,1'-Biphenyl-4-yl)-2-methylthiophen-3-yl)ethanone (3b). Yield $2.45 \mathrm{~g}$ (84\%), light yellow solid, mp 153-154 ${ }^{\circ} \mathrm{C}$. IR $\left(v_{\max }, \mathrm{cm}^{-1}\right): 1801(\mathrm{C}=\mathrm{O}), 1747(\mathrm{C}=\mathrm{C}), 1428 .{ }^{1} \mathrm{H} \mathrm{NMR}(250 \mathrm{MHz}$, $\left.\mathrm{CDCl}_{3}\right): \delta 2.53(\mathrm{~s}, 3 \mathrm{H}, \mathrm{Me}), 2.75(\mathrm{~s}, 3 \mathrm{H}, \mathrm{Me}), 7.37-7.65(\mathrm{~m}, 10 \mathrm{H}$, arom. H, thioph. $\mathrm{H}) .{ }^{13} \mathrm{C} \mathrm{NMR}$ $\left(62.9 \mathrm{MHz}, \mathrm{CDCl}_{3}\right): \delta 16.03 ; 29.68 ; 41.41 ; 124.37 ; 125.84 ; 125.85 ; 126.79 ; 126.80 ; 127.52 ;$ 
127.54; 128.87; 128.88; 132.53; 137.19; 138.92; 140.19; 140.33; 148.14; 193.75. MS (EI, 70 eV), $m / z: 292[\mathrm{M}]^{+}$. Anal. Calcd. for $\mathrm{C}_{19} \mathrm{H}_{16} \mathrm{OS}: \mathrm{C}, 78.05 ; \mathrm{H}, 5.52$. Found: C, 78.13; H, $5.45 \%$.

General procedure for the synthesis of 4a,b. A mixture of $\mathbf{3 a}(0.4 \mathrm{mmol}, 0.1 \mathrm{~g})$ or $\mathbf{3 b}(0.68$ mmol, $0.20 \mathrm{~g})$, sulfur ( $0.8 \mathrm{mmol}, 0.026 \mathrm{~g})$, p-toluenesulfonic acid $(0.14 \mathrm{mmol}, 0.024 \mathrm{~g})$ and morpholine $(1.2 \mathrm{mmol}, 0.11 \mathrm{~g})$ was heated while stirring in an oil bath at $130{ }^{\circ} \mathrm{C}$ for $6 \mathrm{~h}$. The reaction mixture was cooled to ambient temperature and then $5 \mathrm{~mL}$ of $20 \%$ aqueous $\mathrm{NaOH}$ and benzyltriethylammonium chloride (TEBA) (0.02 mmol, $0.005 \mathrm{~g}$,) were added. The reaction mixture was heated at reflux while stirring for $8 \mathrm{~h}$, diluted with $5 \mathrm{~mL}$ of water and cooled to ambient temperature. The solution was filtered and acidified with $10 \%$ aqueous $\mathrm{HCl}$ up to $\mathrm{pH}=$ 6. The precipitate was filtered. The filtrate was further acidified with $10 \%$ aqueous $\mathrm{HCl}$ up to $\mathrm{pH}$ $=2$ and the new portion of precipitate was filtered. The combined precipitate was washed with water, dried and recrystallized from $\mathrm{CCl}_{4}$.

2-(5-(4-Chlorophenyl)-2-methylthiophen-3-yl)acetic acid (4a). Yield $0.68 \mathrm{~g} \mathrm{(64 \% ),} \mathrm{light} \mathrm{yellow}$ solid, mp 134-135 ${ }^{\circ} \mathrm{C}$. IR ( $\left.v_{\max }, \mathrm{cm}^{-1}\right): 3061,2559,1705 .{ }^{1} \mathrm{H}$ NMR $\left(250 \mathrm{MHz}, \mathrm{CDCl}_{3}\right): \delta 2.39$ (s, $3 \mathrm{H}, \mathrm{Me}), 3.55$ (s, 2H, CH ), 7.18 (s, 1H, thioph. H), 7.31-7.39 (d, 2H, arom. H), 7.50-7.54 (d, 2H, arom. H), 10.50-11.60 (br. s, $1 \mathrm{H}, \mathrm{OH}) .{ }^{13} \mathrm{C} \mathrm{NMR} \mathrm{(62.9} \mathrm{MHz,} \mathrm{CDCl}_{3}$ ): $\delta 11.74 ; 33.20 ; 125.69$; $126.13 ; 126.14 ; 128.57 ; 128.58 ; 131.40 ; 132.28 ; 133.12 ; 133.50 ; 138.03 ; 173.52 . \mathrm{MS}$ (EI, $70 \mathrm{eV})$, $m / z: 266[\mathrm{M}]^{+}$. Anal. Calcd. for $\mathrm{C}_{13} \mathrm{H}_{11} \mathrm{ClO}_{2} \mathrm{~S}: \mathrm{C}, 58.54 ; \mathrm{H}, 4.16$. Found: C, 58.43; H, $4.25 \%$.

2-(5-([1,1'-Biphenyl-4-yl)-2-methylthiophen-3-yl)acetic acid (4b). Yield $0.11 \mathrm{~g}$ (53\%), light yellow solid, mp 105-106 ${ }^{\circ} \mathrm{C}$. IR $\left(v_{\max }, \mathrm{cm}^{-1}\right): 3058,2555,1700 .{ }^{1} \mathrm{H} \mathrm{NMR}\left(250 \mathrm{MHz}, \mathrm{CDCl}_{3}\right): \delta$ 2.46 (s, 3H, Me), 3.60 (s, 2H, $\left.\mathrm{CH}_{2}\right), 7.33-7.36(\mathrm{~m}, 1 \mathrm{H}$, thioph. $\mathrm{H}), 7.39-7.71(\mathrm{~m}, 9 \mathrm{H}$, arom. $\mathrm{H})$, 10.50-11.50 (br. s, $1 \mathrm{H}, \mathrm{OH}) .{ }^{13} \mathrm{C} \mathrm{NMR}\left(62.9 \mathrm{MHz}, \mathrm{CDCl}_{3}\right): \delta 21.13 ; 31.75 ; 33.94 ; 125.43 ; 125.44$; $125.69 ; 126.57 ; 126.65 ; 127.34 ; 127.38 ; 127.48 ; 128.88 ; 128.92 ; 131.69 ; 133.44 ; 135.11 ; 139.56$; 140.23; 169.83. MS (EI, $70 \mathrm{eV}), \mathrm{m} / z: 308[\mathrm{M}]^{+}$. Anal. Calcd. for $\mathrm{C}_{19} \mathrm{H}_{16} \mathrm{O}_{2} \mathrm{~S}: \mathrm{C}, 74.00 ; \mathrm{H}, 5.23$. Found: C, 74.13; H, $5.35 \%$.

(2,5-Dimethylthiophen-3-yl)acetic acid (4c). The general procedure above described was applied using 1-(2,5-dimethylthiophen-3-yl)ethanone (10 mmol, $1.54 \mathrm{~g})$. Yield $0.92 \mathrm{~g}$ (54\%), light yellow solid, mp 68-69 ${ }^{\circ} \mathrm{C}$ [lit. ${ }^{22} \mathrm{mp} 69-70{ }^{\circ} \mathrm{C}$ ]. IR $\left(v_{\max }, \mathrm{cm}^{-1}\right): 3068,2565,1705 .{ }^{1} \mathrm{H}$ NMR $(250 \mathrm{MHz}$, $\left.\mathrm{CDCl}_{3}\right) \delta(\mathrm{ppm}): 2.26(\mathrm{~s}, 3 \mathrm{H}, \mathrm{Me}), 2.34(\mathrm{~s}, 3 \mathrm{H}, \mathrm{Me}), 3.39$ (s, 2H, $\left.\mathrm{CH}_{2}\right), 6.56$ (s, 1H, thioph. H), 10.30-11.20 (br. s, $1 \mathrm{H}, \mathrm{OH}$ ). MS (EI, $70 \mathrm{eV}$ ), m/z: $170[\mathrm{M}]^{+}$. Anal. Calcd. for $\mathrm{C}_{8} \mathrm{H}_{10} \mathrm{O}_{2} \mathrm{~S}: \mathrm{C}, 56.44$; H, 5.92. Found: C, 56.43; H, $5.85 \%$.

2-(5-(4-Bromophenyl)-2-methylthiophen-3-yl)acetic acid (4d). The general procedure above described was applied using 2-(5-(4-bromophenyl)-2-methylthiophen-3-yl)-1morpholinoethanethione (10 mmol, $2.95 \mathrm{~g}$ ). Yield $2.15 \mathrm{~g}(69 \%)$, light brown solid, mp 162-163 ${ }^{\circ} \mathrm{C}$ [lit. $\left.{ }^{13} \mathrm{mp} 161-162{ }^{\circ} \mathrm{C}\right]$. IR $\left(v_{\max }, \mathrm{cm}^{-1}\right): 3062,2558,1704 .{ }^{1} \mathrm{H} \mathrm{NMR}\left(250 \mathrm{MHz}, \mathrm{CDCl}_{3}\right): \delta 2.41$ (s, 3H, Me), 3.56 (s, 2H, CH 2$), 7.12$ (s, 1H, thioph. H), 7.21-7.48 (m, 4H, arom. H), 10.60-11.50 (br. s, $1 \mathrm{H}, \mathrm{OH})$. MS (EI, $70 \mathrm{eV}), \mathrm{m} / z: 311[\mathrm{M}]^{+}$. Anal. Calcd. for $\mathrm{C}_{13} \mathrm{H}_{11} \mathrm{BrO}_{2} \mathrm{~S}: \mathrm{C}, 50.17 ; \mathrm{H}, 3.56$. Found: C, 50.08; H, $3.66 \%$. 


\section{Supplementary Material}

${ }^{1} \mathrm{H}$ and ${ }^{13} \mathrm{C}$ spectra for all novel obtained compounds.

\section{Acknowledgements}

This research was financially supported by Grant of President of Russian Federation (No. MK6738.2016.3). E.Sh. and A.D. worked in the framework of the State Order for 2016 No. 00701114-16 PR 0256-2014-0009.

\section{References}

1. Despeyroux, P; Frehel, D.; Schoentjes, B.; Van Dorsselaer, V. FR Patent 2850380 A1 20040730, 2004.

2. Fischer, R.; Dumas, J.; Bretschneider, T.; Gallenkamp, B.; Lieb, F.; Wernthaler, K.; Erdelen, C.; Wachendorff-Neumann, U.; Mencke, N.; Turberg, A. DE Patent 19527190 A1 19960523 , 1996.

3. Minkin, V. I. Russ. Chem. Bull., Int. Ed. 2008, 57, 687. http://dx.doi.org/10.1007/s11172-008-0111-y

4. Krayushkin, M. M.; Lichitskii, B. V.; Pashchenko, D. V.; Antonov, I. A.; Nabatov, B. V., Dudinov, A. A. Russ. J. Org. Chem. 2007, 43, 1357. http://dx.doi.org/10.1134/S1070428007090163

5. Zhang, J.; Zou, Q.; Tian, H. Adv. Mater. 2013, 25, 378. http://dx.doi.org/10.1002/adma.201201521

6. Ma, L.; Wang, Q.; Lu, G.; Chen, R.; Sun, X. Langmuir 2010, 26, 6702. http://dx.doi.org/10.1021/la9040387

7. Aldoshin, S. M.; Yur'eva, E. A.; Sanina, N. A.; Krayushkin, M. M.; Tsyganov, D. V.; Gostev, F. E.; Shelaev, I. V.; Sarkisov, O. M.; Nadtochenko, V. A. Russ. Chem. Bull., Int. Ed. 2011, $60,1118$. http://dx.doi.org/10.1007/s11172-011-0176-x

8. Brown, E. V.; Blanchette, J. A. J. Am. Chem. Soc. 1950, 72, 3414. http://dx.doi.org/10.1021/ja01164a026

9. Bochkov, A. Y.; Krayushkin, M. M.; Yarovenko, V. N.; Barachevsky, V. A.; Beletskaya, I. P.; Traven, V. F. J. Heterocycl. Chem. 2013, 50, 891.

http://dx.doi.org/10.1002/jhet.931 
10. Young, J. R.; Huang, S. X.; Chen, I.; Walsh, T. F.; De Vita, R. J.; Wyvratt Jr., M. J.; Goulet, M. T.; Ren, N.; Lo, J.; Yang, Y. T.; Yudkovitz, J. B.; Cheng, K.; Smith, R. G. Bioorg. Med. Chem. Lett. 2000, 10, 1723 - 1727. http://dx.doi.org/10.1016/S0960-894X(00)00318-8

11. Todd, D. Org. React. 1948, 4, 378. http://dx.doi.org/10.1002/0471264180.or004.08

12. Priebbenow, D. L.; Bolm, C. Chem. Soc. Rev. 2013, 42, 7870. http://dx.doi.org/10.1039/C3CS60154D

13. Shepelenko, E. N.; Makarova, N. I.; Karamov, O. G.; Dubonosov, A. D.; Podshibakin, V. A.; Metelitsa, A. V.; Bren, V. A.; Minkin V. I. Chem. Heterocycl. Compd. 2014, 50, 932. http://dx.doi.org/10.1007/s10593-014-1547-7

14. Alam, M. M.; Adapa, S. R. Synth. Commun. 2003, 33, 59. http://dx.doi.org/10.1081/SCC-120015559

15. Frimm, R.; Fisera, L.; Kovác, J. Collect. Czech. Chem. Commun. 1973, 38, 1809. http://dx.doi.org/10.1135/cccc19731809

16. Polyakov, V. K.; Zaplyuisvechka, Z. P.; Tsukerman, S. V. Chem. Heterocycl. Compd. 1974, 10, 123. http://dx.doi.org/10.1007/BF00475930

17. Stulin, N. V.; Lipkin, A. E.; Kulikova, D. A.; Rudzim, E. A. Pharm. Chem. J. 1975, 9, 702. http://dx.doi.org/10.1007/BF00773291

18. Ohta, A.; Akita, Y.; Ohkuwa, T.; Chiba, M.; Fukunaga, R.; Miyafuji, A.; Nakata, T.; Tani, N.; Aoyagi, Y. Heterocycles 1990, 31, 1951. http://dx.doi.org/10.3987/COM-90-5467

19. Mignani, G.; Leising, F.; Meyrueix, R.; Samson, H. Tetrahedron Lett. 1990, 31, 4743. http://dx.doi.org/10.1016/S0040-4039(00)97721-9

20. Costa, S.P.G.; Batista, R.M.F.; Cardoso, P.; Belsley, M.; Raposo, M.M.M. Eur. J. Org. Chem. 2006, 17, 3938.

21. Kharchenko, V. G.; Voronin, S. P.; Gubina, T. I.; Markushina, I. A.; Oleinik, A. F. Chem. Heterocycl. Compd. 1984, 12, 1321. http://dx.doi.org/10.1007/BF00505950

22. Press, J. B.; McNally, J. J. J. Heterocycl. Chem. 1988, 25, 1571. http://dx.doi.org/10.1002/jhet.5570250559 